Article

\title{
Coupled SU(3)-Structures and Supersymmetry
}

\author{
Anna Fino * and Alberto Raffero * \\ Dipartimento di Matematica “Giuseppe Peano", Università di Torino, via Carlo Alberto 10, \\ 10123 Torino, Italy \\ * Authors to whom correspondence should be addressed; E-Mails: annamaria.fino@unito.it (A.F.); \\ alberto.raffero@unito.it (A.R.); Tel.: +39-011-6702886 (A.F.); +39-011-6702827 (A.R.).
}

Academic Editor: Jonathan Bougie

Received: 13 April 2015 / Accepted: 7 May 2015 / Published: 11 May 2015

\begin{abstract}
We review coupled SU(3)-structures, also known in the literature as restricted half-flat structures, in relation to supersymmetry. In particular, we study special classes of examples admitting such structures and the behavior of flows of $\mathrm{SU}(3)$-structures with respect to the coupled condition.
\end{abstract}

Keywords: supersymmetry; SU(3)-structures; flows

\section{Introduction}

In the physical literature, manifolds endowed with $\mathrm{SU}(3)$-structures have been frequently considered to construct string vacua [1-10].

In this paper, we are mainly interested in the class of $\mathrm{SU}(3)$-structures that are relevant for $\mathcal{N}=1$ compactifications of Type IIA string theory on spaces of the form $\mathrm{AdS}_{4} \times N$, where $\mathrm{AdS}_{4}$ is the four-dimensional anti-de Sitter space and $N$ is a six-dimensional compact smooth manifold. The requirement of $\mathcal{N}=1$ supersymmetry implies the existence of a globally-defined complex spinor on the internal six-manifold $N$. As a consequence, the structure group of $N$ reduces to $\mathrm{SU}(3)$, which is equivalent to the existence on $N$ of an almost Hermitian structure $(h, J, \omega)$ and a complex $(3,0)$-form $\Psi$ of nonzero constant length satisfying some compatibility conditions. As shown in [3], in the case where the two $\mathrm{SU}(3)$-structures are proportional, imposing the Killing spinor equations for four-dimensional $\mathcal{N}=1$ string vacua of Type IIA on $\mathrm{AdS}_{4}$ constrains the intrinsic torsion of the $\mathrm{SU}(3)$-structure to lie in $\mathcal{W}_{1}^{-} \oplus \mathcal{W}_{2}^{-}$. Further constraints on the torsion forms are implied by the Bianchi identities for the background fluxes in the absence of sources. Moreover, all of these constraints are not only necessary, 
but also sufficient to guarantee the existence of solutions. Examples of this kind of solutions were considered, for instance, in $[3,6,11,12]$.

$\mathrm{SU}(3)$-structures whose torsion class is $\mathcal{W}_{1}^{-} \oplus \mathcal{W}_{2}^{-}$are known as coupled $\mathrm{SU}(3)$-structures [13] in the mathematical literature and are characterized by the fact that they are half-flat SU(3)-structures, i.e., both $\psi_{+}:=\Re(\Psi)$ and $\omega \wedge \omega$ are closed forms, having $d \omega$ proportional to $\psi_{+}$. Coupled structures were recently considered in [14-16]. They are of interest, for instance, because their underlying almost Hermitian structure is quasi-Kähler and because they generalize the class of nearly Kähler SU(3)-structures, namely the half-flat structures having $d \omega$ proportional to $\psi_{+}$and $d \psi_{-}$proportional to $\omega \wedge \omega$, where $\psi_{-}:=\Im(\Psi)$.

Up to now, very few examples of manifolds admitting complete nearly Kähler structures are known. In the homogeneous case, there are only finitely many of them by [17], while new complete inhomogeneous examples were recently found on $S^{6}$ and $S^{3} \times S^{3}$ in [18]. Among the remarkable properties of nearly Kähler structures in dimension six, it is worth recalling here that the Riemannian metric $h$ they induce is Einstein, that is its Ricci tensor $\operatorname{Ric}(h)$ is a scalar multiple of $h$. It is then quite natural to ask whether coupled structures inducing Einstein metrics can exist or if requiring that a coupled structure induces an Einstein metric implies that it is actually nearly Kähler. An attempt to find coupled Einstein structures on explicit examples was done in [16], where the existence of invariant coupled Einstein structures was excluded on the compact manifold $S^{3} \times S^{3}$ for $\operatorname{Ad}\left(S^{1}\right)$-invariant Einstein metrics and on all of the six-dimensional Einstein solvmanifolds. While writing this paper, we found out that the work [6], which provides a family of $\mathrm{AdS}_{4}$ vacua in IIA string theory, contains an example of a coupled Einstein structure. This answers the question and can be used to construct examples of $G_{2}$-structures with non-vanishing torsion inducing Einstein and Ricci-flat metrics.

One of the main motivations to study half-flat structures is due to the role they play in the construction of seven-dimensional manifolds with holonomy contained in $G_{2}$. More in detail, by a result of Hitchin [19], on a six-manifold $N$, it is possible to define a flow for $\mathrm{SU}(3)$-structures, the so-called Hitchin flow, which can be solved for any given analytic half-flat structure as the initial condition. A solution to the flow equations consists of a family of half-flat structures depending on a parameter $t \in I \subseteq \mathbb{R}$ and allows one to define a torsionless $G_{2}$-structure on the product manifold $I \times N$. One question that naturally arises is then whether coupled structures, which are in particular half-flat, are preserved by this flow.

A generalization of the Hitchin flow can be introduced considering an $\mathrm{SU}(3)$-structure, not necessarily half-flat, and using it to define a $G_{2}$-structure with torsion on the product manifold $I \times N$. The evolution equations for the differential forms defining the $\mathrm{SU}(3)$-structure can then be obtained by requiring that the intrinsic torsion of the $G_{2}$-structure belongs to a certain torsion class. Of course, the Hitchin flow equations can be recovered as a special case of this generalized flow. This idea was considered, for example, in [20], where the generalized Hitchin flow was used as a tool to study the moduli space of SU(3)-structure manifolds constituting the internal compact space for four-dimensional $\mathcal{N}=\frac{1}{2}$ domain wall solutions of heterotic string theory. In that case, the authors considered the non-compact seven-dimensional manifold defined by combining the direction perpendicular to the domain wall and the internal six-manifold and observed that it is possible to define on it a $G_{2}$-structure whose non-vanishing intrinsic torsion forms can be recovered using the results of $[8,9]$. 
Furthermore, homogeneous spaces admitting coupled structures were used to provide examples of heterotic $\mathcal{N}=\frac{1}{2}$ domain wall solutions with vanishing fluxes in [7], and an attempt to generalize this result in a more general case was done in [9].

The present paper is organized as follows. In Section 2, we review some definitions and properties regarding $\mathrm{SU}(3)$ - and $G_{2}$-structures. In Section 3, we study coupled structures in relation to supersymmetry. In Section 4, we describe some explicit examples, and in Section 5, we study the behavior of flows of SU(3)-structures with respect to the coupled condition.

\section{Review of SU(3)-Structures and $G_{2}$-Structures}

An SU(3)-structure on a six-dimensional smooth manifold $N$ is the data of a Riemannian metric $h$, an orthogonal almost complex structure $J$, a two-form $\omega$ related to $h$ and $J$ via the identity $\omega(\cdot, \cdot)=h(J \cdot, \cdot)$ and a (3,0)-form of nonzero constant length $\Psi=\psi_{+}+i \psi_{-}$, which is compatible with $\omega$, i.e.,

$$
\omega \wedge \Psi=0
$$

and satisfies the normalization condition:

$$
\frac{i}{2}(\Psi \wedge \bar{\Psi})=\frac{2}{3} \omega^{3}=4 d V_{h},
$$

where $d V_{h}$ is the Riemannian volume form of $h$. At each point $p \in N$, there exists an $h$-orthonormal frame $\left(e^{1}, \ldots, e^{6}\right)$ of $T_{p}^{*} N$, called the adapted frame for the $\mathrm{SU}(3)$-structure, such that:

$$
\begin{aligned}
& \omega=e^{12}+e^{34}+e^{56}, \\
& \Psi=\left(e^{1}+i e^{2}\right) \wedge\left(e^{3}+i e^{4}\right) \wedge\left(e^{5}+i e^{6}\right),
\end{aligned}
$$

and whose dual frame $\left(e_{1}, \ldots, e_{6}\right)$ is adapted for $J$, i.e.,

$$
J e_{i}=e_{i+1}, i=1,3,5 \text {. }
$$

Remark 1. Here and hereafter, the notation $e^{i j k \cdots}$ is a shortening for the wedge product $e^{i} \wedge e^{j} \wedge e^{k} \wedge \cdots$. Moreover, we will also use the notation $\theta^{n}$ as a shortening for the wedge product of a differential form $\theta$ by itselffor n-times.

Using the results of [21,22], one can show that an $\mathrm{SU}(3)$-structure actually depends only on the pair $\left(\omega, \psi_{+}\right)$; let us recall briefly how. For each $p \in N$, let $V:=T_{p} N$; denote by $A: \Lambda^{5}\left(V^{*}\right) \rightarrow V \otimes \Lambda^{6}\left(V^{*}\right)$ the canonical isomorphism given by $A(\xi)=v \otimes \Omega$, where $i_{v} \Omega=\xi$, and define for a fixed $\rho \in \Lambda^{3}\left(V^{*}\right)$ :

$$
K_{\rho}: V \rightarrow V \otimes \Lambda^{6}\left(V^{*}\right), \quad K_{\rho}(v)=A\left(\left(i_{v} \rho\right) \wedge \rho\right)
$$

and

$$
\lambda: \Lambda^{3}\left(V^{*}\right) \rightarrow\left(\Lambda^{6}\left(V^{*}\right)\right)^{\otimes 2}, \quad \lambda(\rho)=\frac{1}{6} \operatorname{tr} K_{\rho}^{2} .
$$

If $\lambda(\rho) \neq 0, \sqrt{|\lambda(\rho)|} \in \Lambda^{6}\left(V^{*}\right)$ defines a volume form by choosing the orientation of $V$ for which $\omega^{3}$ is positively oriented. Moreover, whenever $\lambda(\rho)<0$, the following endomorphism defines an almost complex structure:

$$
J_{\rho}:=-\frac{1}{\sqrt{-\lambda(\rho)}} K_{\rho}
$$


An $\mathrm{SU}(3)$-structure on $N$ can then be defined as a pair $\left(\omega, \psi_{+}\right)$, such that the two-form $\omega$ is non degenerate, i.e., $\omega^{3} \neq 0$, the three-form $\psi_{+}$is compatible with $\omega$ and satisfies $\lambda\left(\psi_{+}(p)\right)<0$ for each $p \in N$, the almost complex structure is $J=J_{\psi_{+}}$, the imaginary part of $\Psi$ is given by $\psi_{-}:=J \psi_{+}$, the normalization condition holds and $h(\cdot, \cdot):=\omega(\cdot, J \cdot)$ defines a Riemannian metric.

The intrinsic torsion $\tau$ of an $\mathrm{SU}(3)$-structure is completely determined by the exterior derivatives of $\omega, \psi_{+}, \psi_{-}$, as shown in [23]. More in detail, we have:

$$
\begin{aligned}
& d \omega=-\frac{3}{2} w_{1}^{-} \psi_{+}+\frac{3}{2} w_{1}^{+} \psi_{-}+w_{3}+w_{4} \wedge \omega, \\
& d \psi_{+}=w_{1}^{+} \omega^{2}-w_{2}^{+} \wedge \omega+w_{5} \wedge \psi_{+}, \\
& d \psi_{-}=w_{1}^{-} \omega^{2}-w_{2}^{-} \wedge \omega+J w_{5} \wedge \psi_{+},
\end{aligned}
$$

where $w_{1}^{ \pm} \in C^{\infty}(N), w_{2}^{ \pm} \in \Lambda_{0}^{1,1}(N), w_{3} \in \Lambda_{0}^{2,1}(N), w_{4}, w_{5} \in \Lambda^{1}(N)$ are the intrinsic torsion forms of the $\mathrm{SU}(3)$-structure. It is then possible to divide the $\mathrm{SU}(3)$-structures into classes by seeing which torsion forms vanish. For example, if $\omega, \psi_{+}$and $\psi_{-}$are all closed, then all of the torsion forms vanish, and the manifold $N$ is Calabi-Yau. If all of the torsion forms, but $w_{1}^{-}$, vanish, the $\mathrm{SU}(3)$-structure is said to be nearly Kähler, and we write $\tau \in \mathcal{W}_{1}^{-}$. If both $\psi_{+}$and $\omega^{2}$ are closed, then the torsion forms $w_{1}^{+}, w_{2}^{+}, w_{4}, w_{5}$ vanish, the $\mathrm{SU}(3)$-structure is said to be half-flat, and we write $\tau \in \mathcal{W}_{1}^{-} \oplus \mathcal{W}_{2}^{-} \oplus \mathcal{W}_{3}$. As recently shown in [24], the $\mathrm{SU}(3)$-structures can also be described in terms of a characterizing spinor and the spinorial field equations it satisfies.

In [25], it was shown that the Ricci and the scalar curvature of the metric $h$ induced by an $\mathrm{SU}(3)$-structure can be expressed in terms of the intrinsic torsion forms. In particular, if we consider the projections $E_{1}: \Lambda^{2}(N) \rightarrow \Lambda_{0}^{1,1}(N)$ and $E_{2}: \Lambda^{3}(N) \rightarrow \Lambda_{0}^{2,1}(N)$ given by:

$$
\begin{aligned}
& E_{1}(\beta)=\frac{1}{2}(\beta+J \beta)-\frac{1}{18} *((*(\beta+J \beta)+(\beta+J \beta) \wedge \omega) \wedge \omega) \omega, \\
& E_{2}(\rho)=\rho-\frac{1}{2} *(J \rho \wedge \omega) \wedge \omega-\frac{1}{4} *\left(\rho \wedge \psi_{-}\right) \psi_{+}-\frac{1}{4} *\left(\psi_{+} \wedge \rho\right) \psi_{-},
\end{aligned}
$$

where $*$ is the Hodge operator defined using $h$ and the volume form $d V_{h}$, then the traceless part of the Ricci tensor has the following expression:

$$
\operatorname{Ric}^{0}(h)=\iota^{-1}\left(E_{1}\left(\phi_{1}\right)\right)+\gamma^{-1}\left(E_{2}\left(\phi_{2}\right)\right),
$$

where the two-form $\phi_{1}$ and the three-form $\phi_{2}$ depend on the intrinsic torsion forms and their derivatives and the maps $\iota: S_{+}^{2}(N) \rightarrow \Lambda_{0}^{1,1}(N)$ and $\gamma: S_{-}^{2}(N) \rightarrow \Lambda_{0}^{2,1}(N)$ are (pointwise) su(3)-module isomorphisms (see [25] for details). The Ricci tensor of $h$ can then be recovered from the identity:

$$
\operatorname{Ric}(h)=\frac{1}{6} \operatorname{Scal}(h) h+\operatorname{Ric}^{0}(h) .
$$

Starting from an $\mathrm{SU}(3)$-structure $\left(\omega, \psi_{+}\right)$on a six-manifold $N$, it is possible to construct a $G_{2}$-structure on the seven-manifold $I \times N$, where $I \subseteq \mathbb{R}$ is a connected open interval. Before describing how, we recall that a $G_{2}$-structure on a seven-dimensional manifold $M$ is characterized by the existence of a globally-defined three-form $\varphi$ inducing a Riemannian metric $g_{\varphi}$ and a volume form $d V_{g_{\varphi}}$ given by:

$$
g_{\varphi}(X, Y) d V_{g_{\varphi}}=\frac{1}{6} i_{X} \varphi \wedge i_{Y} \varphi \wedge \varphi
$$


for any pair of vector fields $X, Y \in \mathfrak{X}(M)$. The intrinsic torsion of a $G_{2}$-structure $\varphi$ is completely determined by the exterior derivatives of $\varphi$ and $*_{\varphi} \varphi$, where $*_{\varphi}$ is the Hodge operator defined using the metric $g_{\varphi}$ and the volume form $d V_{g_{\varphi}}$. More in detail, it holds [26]:

$$
\begin{aligned}
d \varphi & =\tau_{0} *_{\varphi} \varphi+3 \tau_{1} \wedge \varphi+*_{\varphi} \tau_{3}, \\
d *_{\varphi} \varphi & =4 \tau_{1} \wedge *_{\varphi} \varphi+\tau_{2} \wedge \varphi,
\end{aligned}
$$

where $\tau_{0} \in C^{\infty}(M), \tau_{1} \in \Lambda^{1}(M), \tau_{2} \in \Lambda_{14}^{2}(M)=\left\{\beta \in \Lambda^{2}(M): *_{\varphi}(\varphi \wedge \beta)=-\beta\right\}, \tau_{3} \in \Lambda_{27}^{3}(M)=$ $\left\{\rho \in \Lambda^{3}(M): \varphi \wedge \rho=0\right.$ and $\left.*_{\varphi} \varphi \wedge \rho=0\right\}$ are the intrinsic torsion forms of the $G_{2}$-structure. Furthermore, in this case, it is possible to classify the $G_{2}$-structures in terms of the non-vanishing torsion forms. For example, if $\varphi$ is both closed and co-closed, then all of the torsion forms vanish, $\operatorname{Hol}\left(g_{\varphi}\right) \subseteq G_{2}$ and the $G_{2}$-structure is called parallel. If $\varphi$ is a closed form, then all of the torsion forms, but $\tau_{2}$, vanish, and the $G_{2}$-structure is said to be calibrated. If the only non-vanishing torsion forms are $\tau_{1}$ and $\tau_{2}$, then at least locally, the metric $g_{\varphi}$ is conformally equivalent to the metric induced by a calibrated $G_{2}$-structure, and the $G_{2}$-structure is called locally-conformal calibrated. If the only vanishing torsion form is $\tau_{2}$, then the $G_{2}$-structure is said to be integrable. In this case, there exists a unique affine connection with totally skew-symmetric torsion preserving the $G_{2}$-structure by [27].

Consider now $\left(\omega, \psi_{+}\right)$and two smooth functions $F: I \rightarrow \mathbb{C}-\{0\}$ and $G: I \rightarrow \mathbb{R}^{+}$; the following three-form defines a $G_{2}$-structure on $I \times N$ [28]:

$$
\varphi=\Re\left(F^{3} \Psi\right)+G|F|^{2} \omega \wedge d t
$$

where $t$ is the coordinate on $I$. Moreover, we have:

$$
\begin{aligned}
g_{\varphi} & =G^{2} d t^{2}+|F|^{2} h, \\
d V_{g_{\varphi}} & =G|F|^{6} d t \wedge d V_{h}, \\
*_{\varphi} \varphi & =G \Im\left(F^{3} \Psi\right) \wedge d t+\frac{1}{2}|F|^{4} \omega^{2} .
\end{aligned}
$$

For some particular choices of the interval $I$ and the functions $F$ and $G$, we obtain the following remarkable manifolds:

- the cylinder $C y l(N)$ with metric $d t^{2}+h$, if $I=\mathbb{R}$ and $G, F \equiv 1$,

- the cone $C(N)$ with the metric $d t^{2}+t^{2} h$, if $I=\mathbb{R}^{+}, G \equiv 1$ and $F(t)=t$,

- the sin-cone $S C(N)$ with the metric $d t^{2}+\sin ^{2}(t) h$, if $I=(0, \pi), G \equiv 1$ and $F(t)=\sin (t) e^{i \frac{t}{3}}$.

Observe that with the choice $G \equiv 1$, the manifold $I \times N$ with metric $d t^{2}+|F|^{2} h$ is the warped product of $I$ and $N$ with warping function $|F|$. Using the expression of the Ricci tensor of the warped product metric [29], it is possible to show the following general properties (see also [30]).

Proposition 1. Let $\left(M^{m}, g\right)$ be a Riemannian manifold of dimension $m$. Then, the cone metric $d t^{2}+t^{2} g$ is Ricci-flat if and only if the metric $g$ is Einstein with $\operatorname{Ric}(g)=(m-1) g$.

Proposition 2. Let $\left(M^{m}, g\right)$ be a Riemannian manifold of dimension $m$ with Einstein metric $g$, such that $\operatorname{Ric}(g)=(m-1) g$. Then, the sin-cone metric $d t^{2}+\sin ^{2}(t) h$ is Einstein with Einstein constant $m$. 


\section{Coupled Structures and Supersymmetry}

In [3], the authors considered the problem of finding necessary and sufficient conditions for $\mathcal{N}=1$ compactification of (massive) IIA supergravity to four-dimensional anti-de Sitter space on manifolds endowed with an $\mathrm{SU}(3)$-structure. As a result, they obtained a set of constraints that the intrinsic torsion forms of the $\mathrm{SU}(3)$-structure $\left(\omega, \psi_{+}\right)$on the internal manifold have to satisfy; we recall them here briefly. Supersymmetry equations and the Bianchi identities constrain the intrinsic torsion to lie in the space $\mathcal{W}_{1}^{-} \oplus \mathcal{W}_{2}^{-}$, i.e., the only non-vanishing intrinsic torsion forms are $w_{1}^{-}$and $w_{2}^{-}$. Furthermore, in absence of sources, the Bianchi identities provide a further constraint on the exterior derivative of $w_{2}^{-}$:

$$
d w_{2}^{-} \propto \psi_{+}
$$

and the norms of $w_{1}^{-}$and $w_{2}^{-}$have to satisfy the following inequality [12]:

$$
3\left(w_{1}^{-}\right)^{2} \geq\left|w_{2}^{-}\right|^{2}
$$

where $|\cdot|$ denotes the norm with respect to the metric $h$ induced by the $\mathrm{SU}(3)$-structure. In the massless limit, the solutions reduce to $\mathrm{AdS}_{4} \times N, N$ being a compact six-manifold endowed with an $\mathrm{SU}(3)$-structure with torsion in $\mathcal{W}_{1}^{-} \oplus \mathcal{W}_{2}^{-}$and for which Equation (4) holds. Moreover, it was observed in [12] that Equations (4) and (5) can be relaxed in the presence of sources.

It is then worth studying from the mathematical point of view the properties of this kind of $\mathrm{SU}(3)$-structures. In what follows, we suppose that the manifold $N$ is connected.

First of all, we recall that $\mathrm{SU}(3)$-structures having torsion class $\mathcal{W}_{1}^{-} \oplus \mathcal{W}_{2}^{-}$are known as coupled structures [13] or restricted half-flat structures [10] in the literature. They can be defined as the subclass of half-flat structures having $w_{3} \equiv 0$. In this case, $d \omega$ is proportional to $\psi_{+}$; the intrinsic torsion form $w_{1}^{-}$ is constant [16] and has to be nonzero if we want the intrinsic torsion $\tau$ to belong to the class $\mathcal{W}_{1}^{-} \oplus \mathcal{W}_{2}^{-}$. Thus, if we let $c:=-\frac{3}{2} w_{1}^{-}$, we have:

$$
\begin{aligned}
d \omega & =c \psi_{+}, \\
d \psi_{+} & =0 \\
d \psi_{-} & =-\frac{2}{3} c \omega^{2}-w_{2}^{-} \wedge \omega .
\end{aligned}
$$

The two-form $w_{2}^{-}$lies in the space $\Lambda_{0}^{1,1}(N)$; therefore, it satisfies the following properties:

$$
\begin{aligned}
w_{2}^{-} \wedge \omega^{2} & =0, \\
w_{2}^{-} \wedge \psi_{ \pm} & =0, \\
w_{2}^{-} \wedge \omega & =-* w_{2}^{-} .
\end{aligned}
$$

Using Equation (9) and the expression of $d \psi_{-}$, it is easy to show that the two-form $w_{2}^{-}$is co-closed, that is $\delta w_{2}^{-}=* d * w_{2}^{-}=0$.

Remark 2. Observe that if a manifold admits a coupled structure $\left(\omega, \psi_{+}\right)$with coupled constant $c \in$ $\mathbb{R}-\{0\}$, such that $d \omega=c \psi_{+}$, then one can choose a nonzero real constant $r$, define $\tilde{\omega}:=r^{2} \omega$, $\tilde{\psi}_{+}:=r^{3} \psi_{+}$and obtain a new coupled structure $\left(\tilde{\omega}, \tilde{\psi}_{+}\right)$with coupled constant $\tilde{c}=\frac{c}{r}$. In particular, it is always possible to find a coupled structure having a positive coupled constant. 
From the results of [25], we have that the scalar curvature of the metric $h$ induced by a coupled structure is given by:

$$
\operatorname{Scal}(h)=\frac{15}{2}\left(w_{1}^{-}\right)^{2}-\frac{1}{2}\left|w_{2}^{-}\right|^{2}
$$

Moreover, the forms $\phi_{1}$ and $\phi_{2}$ appearing in the traceless part of the Ricci tensor are:

$$
\begin{aligned}
& \phi_{1}=\frac{1}{4} *\left(w_{2}^{-} \wedge w_{2}^{-}\right)+\frac{1}{4} \delta\left(w_{1}^{-} \psi_{+}\right), \\
& \phi_{2}=-2 * J\left(d w_{2}^{-}\right) .
\end{aligned}
$$

Let us now focus on Equation (4). It forces the proportionality constant between $d w_{2}^{-}$and $\psi_{+}$to satisfy the following result.

Proposition 3. Let $\left(\omega, \psi_{+}\right)$be a coupled $\mathrm{SU}(3)$-structure and suppose that d $w_{2}^{-}$is proportional to $\psi_{+}$. Then it holds:

$$
d w_{2}^{-}=-\frac{\left|w_{2}^{-}\right|^{2}}{4} \psi_{+}
$$

Moreover, the norm of $w_{2}^{-}$is constant.

Proof. First of all, observe that if $d w_{2}^{-}=k \psi_{+}$for some function $k \in C^{\infty}(N)$, then $k$ has to be constant. Indeed, taking the exterior derivatives of both sides, we get:

$$
d k \wedge \psi_{+}=0
$$

which implies $d k=0$. Now, suppose that $d w_{2}^{-}=k \psi_{+}$. Then, starting from $w_{2}^{-} \wedge \psi_{-}=0$, taking the exterior derivatives of both sides and using the previous identities, we have:

$$
\begin{aligned}
0 & =d w_{2}^{-} \wedge \psi_{-}+w_{2}^{-} \wedge d \psi_{-}=k \psi_{+} \wedge \psi_{-}-w_{2}^{-} \wedge w_{2}^{-} \wedge \omega \\
& =\frac{2}{3} k \omega^{3}+w_{2}^{-} \wedge * w_{2}^{-}=\frac{2}{3} k \omega^{3}+\left|w_{2}^{-}\right|^{2} * 1 \\
& =\frac{2}{3} k \omega^{3}+\left|w_{2}^{-}\right|^{2} \frac{1}{6} \omega^{3} .
\end{aligned}
$$

Thus, $k=-\frac{\left|w_{2}^{-}\right|^{2}}{4}$. From the observation made at the beginning of the proof, we also get that $\left|w_{2}^{-}\right|$ is constant.

From Proposition 3 and the fact that $w_{1}^{-}$is constant, we obtain the following constraint.

Proposition 4. Let $\left(\omega, \psi_{+}\right)$be a coupled $\mathrm{SU}(3)$-structure, such that d $w_{2}^{-}$is proportional to $\psi_{+}$. Then, the scalar curvature of the metric induced by the coupled structure is constant.

Proof. Consider Equation (10) of the scalar curvature of $h$, and conclude using the fact that both $w_{1}^{-}$ and $\left|w_{2}^{-}\right|$are constant.

Consider now Equation (5); this implies a further constraint on the scalar curvature.

Proposition 5. Let $\left(\omega, \psi_{+}\right)$be a coupled SU(3)-structure whose non-vanishing intrinsic torsion forms satisfy $3\left(w_{1}^{-}\right)^{2} \geq\left|w_{2}^{-}\right|^{2}$. Then, the scalar curvature of the metric induced by the coupled structure is positive. Moreover, it is also constant if $d w_{2}^{-}$is proportional to $\psi_{+}$. 
Proof. Using the expression of the scalar curvature of a coupled structure and the inequality $3\left(w_{1}^{-}\right)^{2} \geq$ $\left|w_{2}^{-}\right|^{2}$, we get:

$$
\operatorname{Scal}(h)=\frac{15}{2}\left(w_{1}^{-}\right)^{2}-\frac{1}{2}\left|w_{2}^{-}\right|^{2} \geq 2\left|w_{2}^{-}\right|^{2}>0 .
$$

Moreover, if $d w_{2}^{-}$is proportional to $\psi_{+}$, then the scalar curvature is constant by Proposition 4 .

It is also easy to characterize the coupled structures having $d w_{2}^{-}$proportional to $\psi_{+}$and inducing an Einstein metric:

Proposition 6. Let $\left(\omega, \psi_{+}\right)$be a coupled $\mathrm{SU}(3)$-structure, such that d $w_{2}^{-}$is proportional to $\psi_{+}$. Then, the induced metric $h$ is Einstein if and only if the following identity holds:

$$
*\left(w_{2}^{-} \wedge w_{2}^{-}\right)=w_{1}^{-} w_{2}^{-}-\frac{\left|w_{2}^{-}\right|^{2}}{3} \omega .
$$

Proof. Recall that a Riemannian metric $h$ is Einstein if and only if $\operatorname{Ric}^{0}(h)=0$. We know that:

$$
\operatorname{Ric}^{0}(h)=\iota^{-1}\left(E_{1}\left(\phi_{1}\right)\right)+\gamma^{-1}\left(E_{2}\left(\phi_{2}\right)\right),
$$

where $\phi_{1}$ and $\phi_{2}$ for a coupled structure are given in Equation (11). Now, using the fact that $d w_{2}^{-}$is proportional to $\psi_{+}$, one gets that also $\phi_{2}$ is proportional to $\psi_{+}$. Thus, $E_{2}\left(\phi_{2}\right)=0$, since $\psi_{+}$belongs to a subspace of $\Lambda^{3}(N)$, which is disjoint from $\Lambda_{0}^{2,1}(N)$. Moreover,

$$
\phi_{1}=\frac{1}{4} *\left(w_{2}^{-} \wedge w_{2}^{-}\right)-\frac{1}{2}\left(w_{1}^{-}\right)^{2} \omega-\frac{1}{4} w_{1}^{-} w_{2}^{-}
$$

and

$$
E_{1}\left(\phi_{1}\right)=\frac{1}{4} *\left(w_{2}^{-} \wedge w_{2}^{-}\right)-\frac{1}{4} w_{1}^{-} w_{2}^{-}+\frac{1}{12}\left|w_{2}^{-}\right|^{2} \omega
$$

Therefore, $\operatorname{Ric}^{0}(h)=\iota^{-1}\left(E_{1}\left(\phi_{1}\right)\right)$ is zero if and only if $E_{1}\left(\phi_{1}\right)$ is zero, and from this, the assertion follows.

\section{Examples}

In this section, we examine some examples of six-manifolds admitting an $\mathrm{SU}(3)$-structure satisfying (all or in part) the properties discussed in Section 3.

\subsection{Nilmanifolds}

We recall here the definition of a nilmanifold and some useful properties.

Definition 7. Let $G$ be a connected, simply-connected, nilpotent Lie group and $\Gamma$ a cocompact discrete subgroup. The compact quotient manifold $G / \Gamma$ is called nilmanifold.

In the general case, every left invariant tensor on $G$ passes to the quotient defining an invariant tensor on the nilmanifold $G / \Gamma$. Moreover, all of the 34 six-dimensional nilpotent Lie algebras existing up to isomorphisms [31] satisfy the following result. 
Proposition 8 ([32]). Let $\mathfrak{g}$ be a nilpotent Lie algebra and suppose there exists a basis of it, such that the structure constants determined with respect to this basis are rational numbers. Then, if $G$ denotes the simply connected nilpotent Lie group whose Lie algebra is $\mathfrak{g}$, there exists a discrete subgroup $\Gamma$ of $G$, such that $G / \Gamma$ is a nilmanifold.

It then follows that there is a $1-1$ correspondence between invariant $\mathrm{SU}(3)$-structures $\left(\omega, \psi_{+}\right)$on a nilmanifold and pairs $\left(\omega, \psi_{+}\right)$defining an $\mathrm{SU}(3)$-structure on its nilpotent Lie algebra. This allows one to work only with $\mathrm{SU}(3)$-structures defined on nilpotent Lie algebras.

Since every nilpotent Lie group is solvable, the following result by Milnor holds in the case we are considering.

Theorem 9 ([33]). Let $G$ be a solvable Lie group. Then, every left invariant metric on $G$ is either flat or has strictly negative scalar curvature.

In particular, if a nilpotent Lie algebra is endowed with an inner product $h$, then $\operatorname{Scal}(h)$ is non-positive. As a consequence, using Proposition 5, it is immediate to show that:

Proposition 10. There are no six-dimensional nilmanifolds admitting an invariant coupled structure satisfying the condition $3\left(w_{1}^{-}\right)^{2} \geq\left|w_{2}^{-}\right|^{2}$.

Thus, we can only look for nilpotent Lie algebras endowed with a coupled structure $\left(\omega, \psi_{+}\right)$having $d w_{2}^{-}$proportional to $\psi_{+}$. In [14], we showed that among the 34 non-isomorphic six-dimensional nilpotent Lie algebras, there are only two of them admitting a coupled structure; we recall the result here.

Proposition 11 ([14]). Let $\mathfrak{g}$ be a six-dimensional, non-abelian, nilpotent Lie algebra endowed with a coupled $\mathrm{SU}(3)$-structure $\left(\omega, \psi_{+}\right)$. Then, $\mathfrak{g}$ is isomorphic to one of the following nilpotent Lie algebras:

$$
\begin{aligned}
\mathfrak{I} & =\left(0,0,0,0, e^{14}+e^{23}, e^{13}-e^{24}\right), \\
\mathfrak{N} & =\left(0,0,0, e^{13}, e^{14}+e^{23}, e^{13}-e^{15}-e^{24}\right) .
\end{aligned}
$$

Remark 3. Recall that the notation $\mathfrak{I}=\left(0,0,0,0, e^{14}+e^{23}, e^{13}-e^{24}\right)$ means that there exists a basis of one-forms $\left(e^{1}, \ldots, e^{6}\right)$ for $\mathfrak{I}^{*}$, such that $d e^{1}=0, d e^{2}=0, d e^{3}=0, d e^{4}=0, d e^{5}=e^{14}+e^{23}, d e^{6}=$ $e^{13}-e^{24}$, where $d$ is the Chevalley-Eilenberg differential.

Observe that the two Lie algebras $\mathfrak{I}$ and $\mathfrak{N}$ are isomorphic respectively to the Lie algebras labeled by $\mathfrak{n}_{28}$ and $\mathfrak{n}_{9}$ in the work [14]. Here, they are given with different structure equations, since in both cases, the frame $\left(e^{1}, \ldots, e^{6}\right)$ is an adapted frame for the coupled $\mathrm{SU}(3)$-structure. We emphasize some properties of these coupled structures in the following examples.

Example 1. $\mathfrak{I}=\left(0,0,0,0, e^{14}+e^{23}, e^{13}-e^{24}\right)$ is (isomorphic to) the well-known Iwasawa-Lie algebra, which is the Lie algebra of the six-dimensional nilmanifold known in the literature as the Iwasawa manifold (see, for instance, [34] for the definition). Since the frame $\left(e^{1}, \ldots, e^{6}\right)$ is adapted, we have that the pair

$$
\begin{aligned}
& \omega=e^{12}+e^{34}+e^{56} \\
& \psi_{+}=e^{135}-e^{146}-e^{236}-e^{245}
\end{aligned}
$$


defines an $\mathrm{SU}(3)$-structure on $\mathfrak{I}$. In this case $d \omega=-\psi_{+}$, and the non-vanishing intrinsic torsion forms are:

$$
\begin{aligned}
& w_{1}^{-}=\frac{2}{3}, \\
& w_{2}^{-}=-\frac{4}{3} e^{12}-\frac{4}{3} e^{34}+\frac{8}{3} e^{56} .
\end{aligned}
$$

It is easy to check that Equation (4) is satisfied:

$$
d w_{2}^{-}=-\frac{8}{3} \psi_{+}
$$

and that $-\frac{1}{4}\left|w_{2}^{-}\right|^{2}=-\frac{8}{3}$, as we expected from Proposition 3. Finally, the scalar curvature of the metric $h$ induced by the coupled structure is $\operatorname{Scal}(h)=-2$.

Example 2. Consider the Lie algebra $\mathfrak{N}=\left(0,0,0, e^{13}, e^{14}+e^{23}, e^{13}-e^{15}-e^{24}\right)$. Since the frame $\left(e^{1}, \ldots, e^{6}\right)$ is adapted, we have that the pair

$$
\begin{aligned}
& \omega=e^{12}+e^{34}+e^{56} \\
& \psi_{+}=e^{135}-e^{146}-e^{236}-e^{245}
\end{aligned}
$$

defines an $\mathrm{SU}(3)$-structure on $\mathfrak{N}$. Moreover, $d \omega=-\psi_{+}$, and the non-vanishing intrinsic torsion forms are:

$$
\begin{aligned}
& w_{1}^{-}=\frac{2}{3} \\
& w_{2}^{-}=-\frac{4}{3} e^{12}-\frac{4}{3} e^{34}+e^{36}-e^{45}+\frac{8}{3} e^{56} .
\end{aligned}
$$

In this case d $w_{2}^{-}$is not proportional to $\psi_{+}$, and the scalar curvature of the metric $h$ induced by the coupled structure is $\operatorname{Scal}(h)=-3$.

The fact that the Iwasawa manifold admits an invariant coupled structure was also observed in [3], where the authors wrote that it was the unique nilmanifold admitting a coupled structure they knew. Proposition 11 states that, up to isomorphisms, there are only two non-abelian nilpotent Lie algebras admitting a coupled structure, one of which is the Iwasawa. Moreover, as observed in Example 1, the coupled structure on the Iwasawa-Lie algebra satisfies Equation (4), i.e., dw $w_{2}^{-}$is proportional to $\psi_{+}$. Thus, it is a natural question to ask whether $\mathfrak{N}$ admits a coupled structure satisfying Equation (4) or not. In [11], the authors looked for the possible nilmanifolds admitting an invariant coupled structure satisfying Equation (4) and concluded that a systematic scan of all of the possible six-dimensional nilmanifolds yields two possibilities: the six-torus and the Iwasawa manifold. The six-torus has abelian Lie algebra, so it is not considered in Proposition 11. Moreover, the intrinsic torsion forms of any invariant SU(3)-structure defined on it are all zero. Anyway, this result seems to answer negatively our question, and we can prove this is actually what happens.

Proposition 12. There are no coupled $\mathrm{SU}(3)$-structures on $\mathfrak{N}$ for which the exterior derivative of the intrinsic torsion form $w_{2}^{-}$is proportional to $\psi_{+}$. 
Proof. The idea is to describe all of the possible coupled structures on $\mathfrak{N}$ and to see if there exists one of these whose intrinsic torsion form $w_{2}^{-}$satisfies the required condition. Let us start considering a generic two-form $\omega$ on $\mathfrak{N}$; we can write it as:

$$
\omega=\sum_{1 \leq i<j \leq 6} \omega_{i j} e^{i j}
$$

where $\omega_{i j}$ are real numbers. We may think of the 15 -tuple $\left(\omega_{12}, \ldots, \omega_{56}\right)=:\left(\omega_{i j}\right)$ as a point in the affine space $\mathbb{A}_{\mathbb{R}}^{15}-\{0\}$. The homogeneous polynomial $P$ of degree three in the unknowns $\omega_{i j}$ appearing as a coefficient of $e^{123456}$ in the expression of $\omega^{3}$ has to be non-vanishing; this gives a first constraint for $\left(\omega_{i j}\right)$. Since we want a coupled structure, we consider a three-form $\psi_{+}$on $\mathfrak{N}$ given by $\psi_{+}=c d \omega$, for some nonzero real number $c$. Assuming:

$$
\lambda\left(\psi_{+}\right)=-4 c^{4} \omega_{56}^{2}\left(\omega_{36} \omega_{56}-\omega_{45} \omega_{56}-\omega_{46}^{2}+\omega_{56}^{2}\right)<0,
$$

that is $\omega_{56} \neq 0$ and $B:=\omega_{36} \omega_{56}-\omega_{45} \omega_{56}-\omega_{46}^{2}+\omega_{56}^{2}>0$, we can compute the almost complex structure $J$ induced by the stable form $\psi_{+}$. Now, we change the basis from $\left(e_{1}, \ldots, e_{6}\right)$ to a basis $\left(E_{1}, \ldots, E_{6}\right)$, which is adapted for $J$. To do this, it suffices to define $E_{i}=e_{i}$ and $E_{i+1}=J e_{i}$ for $i=1,3,5$. With respect to $\left(E_{1}, \ldots, E_{6}\right)$, the matrix associated to $J$ is skew-symmetric with non-vanishing entries given by $J^{2}{ }_{1}=1=J^{4}{ }_{3}=J^{6}{ }_{5}$. We can then compute the new structure equations with respect to the dual basis $\left(E^{1}, \ldots, E^{6}\right)$, obtaining:

$$
\begin{aligned}
& d E^{i}=0, \quad i=1,2,3 \\
& d E^{4}=\frac{\omega_{56}}{\sqrt{B}} E^{13}, \\
& d E^{5}=-\frac{\omega_{46}}{\omega_{56}} E^{13}+\frac{\sqrt{B}}{\omega_{56}}\left(E^{14}+E^{23}\right), \\
& d E^{6}=-\frac{\omega_{26}}{\omega_{56}} E^{12}-\frac{\omega_{46}}{\omega_{56}} E^{14}-\frac{\omega_{36} \omega_{56}+\omega_{45} \omega_{56}-\omega_{46}^{2}-\omega_{56}^{2}}{\omega_{56} \sqrt{B}} E^{13}-\frac{\omega_{56}}{\sqrt{B}} E^{15}-\frac{\sqrt{B}}{\omega_{56}} E^{24} .
\end{aligned}
$$

Moreover, we have:

$$
\begin{aligned}
& \psi_{+}=-c \frac{B}{\omega_{56}}\left(E^{135}-E^{146}-E^{236}-E^{245}\right), \\
& \psi_{-}=-c \frac{B}{\omega_{56}}\left(E^{136}+E^{145}+E^{235}-E^{246}\right) .
\end{aligned}
$$

We can write $\omega$ with respect to the new basis and impose it to be of type $(1,1)$ with respect to $J$, obtaining three equations in the variables $\omega_{i j}$, which can be solved under the constraint $\lambda\left(\psi_{+}\right)<0$. We can then consider the symmetric matrix $H$ associated with $h(\cdot, \cdot)=\omega(\cdot, J \cdot)$ with respect to the basis $\left(E_{1}, \ldots, E_{6}\right)$ and denote by $\mathcal{P} \subset \mathbb{A}_{\mathbb{R}}^{15}$ the set on which it is positive definite. One can check that $P \neq 0$ when $\left(\omega_{i j}\right) \in \mathcal{P}$. Now, if we let $\left(\omega_{i j}\right)$ vary in the (non-empty) set $\mathcal{Q}:=\mathcal{P} \cap\left\{\left(\omega_{i j}\right): \lambda\left(\psi_{+}\right)<0\right\}$, we have all of the possible non-normalized coupled $\mathrm{SU}(3)$-structures on $\mathfrak{N}$. The intrinsic torsion form $w_{1}^{-}$ is always $-\frac{2}{3 c}$, while $w_{2}^{-}$can be computed from its defining properties and the expression of $d \psi_{-}$. We are interested in the coupled structures having $w_{2}^{-}$, such that $d w_{2}^{-}$is proportional to $\psi_{+}$. Thus, we can start with a generic two-form $w$ of type $(1,1)$ with respect to $J$ and write it as:

$$
\begin{aligned}
w= & w_{12} E^{12}+w_{34} E^{34}+w_{56} E^{56}+w_{13}\left(E^{13}+E^{24}\right)+w_{14}\left(E^{14}-E^{23}\right)+w_{15}\left(E^{15}+E^{26}\right) \\
& w_{16}\left(E^{16}-E^{25}\right)+w_{35}\left(E^{35}+E^{46}\right)+w_{36}\left(E^{36}-E^{45}\right),
\end{aligned}
$$


where $w_{i j}$ are real numbers. Then, we have to impose that $w$ is primitive $\left(w \wedge \omega^{2}=0\right)$ and fulfills

$$
d \psi_{-}=-\frac{2}{3 c} \omega^{2}-w \wedge \omega
$$

and that $d w$ is proportional to $\psi_{+}$. The last condition gives rise to a set of polynomial equations in the variables $w_{i j}$ with coefficients depending on $\omega_{i j}$, which can be solved in $\mathcal{Q}$. The condition on $d \psi_{-}$gives 13 equations of the same kind as before; we can solve four of them, namely those obtained comparing the coefficients of $E^{3456}, E^{2356}, E^{1256}, E^{2345}$, but then we get that some of the remaining equations can be solved only if $c=0$ or $\lambda\left(\psi_{+}\right)=0$. The assertion is then proven.

The previous results can be summarized as follows.

Proposition 13. Let $\mathfrak{g}$ be a six-dimensional, non-abelian, nilpotent Lie algebra endowed with a coupled $\mathrm{SU}(3)$-structure $\left(\omega, \psi_{+}\right)$having d $w_{2}^{-}$proportional to $\psi_{+}$. Then, $\mathfrak{g}$ is isomorphic to the Iwasawa-Lie algebra.

\subsection{Twistor Spaces}

In the work [6], it was observed that on the twistor space $Z$ over a self-dual Einstein four-manifold $\left(M^{4}, g\right)$, there exists a coupled structure. Moreover, for a suitable value of the scalar curvature of $g$, the metric induced by this structure is Einstein.

Recall that given a four-dimensional, oriented Riemannian manifold $\left(M^{4}, g\right)$, the set of positive, orthogonal almost complex structures on $M^{4}$ forms a smooth manifold $Z$ called the twistor space of $M^{4}$, which can be viewed as the two-sphere bundle $\pi: Z \rightarrow M^{4}$ consisting of the unit -1 eigenvectors of the Hodge operator acting on $\Lambda^{2} T M^{4}$ [35].

On $Z$, it is possible to define two almost complex structures (see, for example, [36]), one of which is never integrable, as shown in [37]. Let us denote it by $J$.

When the metric $g$ is self-dual and Einstein, Xu showed in [38] that on $(Z, J)$, there exists a basis of $(1,0)$-forms $\varepsilon^{1}, \varepsilon^{2}, \varepsilon^{3}$, such that the first structure equations are:

$$
d\left(\begin{array}{l}
\varepsilon^{1} \\
\varepsilon^{2} \\
\varepsilon^{3}
\end{array}\right)=-\left(\begin{array}{cc}
\alpha & 0 \\
0 & -\operatorname{tr}(\alpha)
\end{array}\right) \wedge\left(\begin{array}{l}
\varepsilon^{1} \\
\varepsilon^{2} \\
\varepsilon^{3}
\end{array}\right)+\left(\begin{array}{c}
\overline{\varepsilon^{2} \wedge \varepsilon^{3}} \\
\overline{\varepsilon^{3} \wedge \varepsilon^{1}} \\
\sigma \overline{\varepsilon^{1} \wedge \varepsilon^{2}}
\end{array}\right)
$$

where $\alpha$ is a $2 \times 2$ skew-Hermitian matrix of one-forms and $\sigma:=\frac{\operatorname{Scal}(g)}{24}$. Using these, it is easy to show that the following pair of forms defines a coupled $\mathrm{SU}(3)$-structure on $Z$ [6]:

$$
\begin{aligned}
& \omega=\frac{i}{2}\left(\varepsilon^{1} \wedge \overline{\varepsilon^{1}}+\varepsilon^{2} \wedge \overline{\varepsilon^{2}}+\varepsilon^{3} \wedge \overline{\varepsilon^{3}}\right), \\
& \Psi=i\left(\varepsilon^{1} \wedge \varepsilon^{2} \wedge \varepsilon^{3}\right) .
\end{aligned}
$$

Observe that $J$ is the almost complex structure induced by $\Re(\Psi)$ and that the metric induced by $\omega$ and $J$ takes the following form:

$$
h=\varepsilon^{1} \odot \overline{\varepsilon^{1}}+\varepsilon^{2} \odot \overline{\varepsilon^{2}}+\varepsilon^{3} \odot \overline{\varepsilon^{3}} .
$$


Moreover, the non-vanishing intrinsic torsion forms have the following expressions:

$$
\begin{aligned}
& w_{1}^{-}=\frac{2}{3}(\sigma+2) \\
& w_{2}^{-}=-\frac{2}{3} i(\sigma-1)\left(\varepsilon^{1} \wedge \overline{\varepsilon^{1}}+\varepsilon^{2} \wedge \overline{\varepsilon^{2}}-2 \varepsilon^{3} \wedge \overline{\varepsilon^{3}}\right),
\end{aligned}
$$

$d w_{2}^{-}$is proportional to $\psi_{+}$

$$
d w_{2}^{-}=-\frac{8}{3}(\sigma-1)^{2} \psi_{+}
$$

and $3\left(w_{1}^{-}\right)^{2} \geq\left|w_{2}^{-}\right|^{2}$ if and only if $\frac{10-6 \sqrt{2}}{7} \leq \sigma \leq \frac{10+6 \sqrt{2}}{7}$.

We can consider a local frame $\left(e^{1}, \ldots, e^{6}\right)$ for $\Lambda^{1}(Z)$, such that $\varepsilon^{1}=e^{1}+i e^{2}, \varepsilon^{2}=e^{3}+i e^{4}$, $\varepsilon^{3}=e^{5}+i e^{6}$, and compute the Ricci curvature of the metric induced by the coupled structure using the results of [25]. What we get is that the scalar curvature of $h$ is:

$$
\operatorname{Scal}(h)=-2 \sigma^{2}+24 \sigma+8
$$

and the traceless part of the Ricci tensor of $h$ with respect to the considered frame has the following form:

$$
\operatorname{Ric}^{0}(h)=-\frac{2}{3}(\sigma-1)(\sigma-2) \operatorname{diag}(1,1,1,1,-2,-2) .
$$

Thus, the metric $h$ is Einstein if and only if $\sigma=1$ or $\sigma=2$, that is if and only if the scalar curvature of $g$ is 24 or 48 , respectively. In the first case, the coupled structure is actually nearly Kähler, since $w_{2}^{-}=0$, while in the second case, we get an example of a coupled SU(3)-structure inducing an Einstein metric. More in detail, the latter has the following non-vanishing intrinsic torsion forms:

$$
\begin{aligned}
& w_{1}^{-}=\frac{8}{3}, \\
& w_{2}^{-}=-\frac{4}{3}\left(e^{12}+e^{34}-2 e^{56}\right) .
\end{aligned}
$$

In particular, the coupled constant is $c=-4$, and the scalar curvature is $\operatorname{Scal}(h)=48$. Moreover, the characterization given in Proposition 6 is satisfied by this example.

Recall that when $\operatorname{Scal}(g)>0$, a compact, self-dual, Einstein four-manifold $\left(M^{4}, g\right)$ is isometric either to $S^{4}$ or to $\mathbb{C P}^{2}$ with their canonical metrics (Theorem 13.30 in [39]); thus, $Z$ is either $\mathbb{C P}^{3}$ or the flag manifold $\mathrm{SU}(3) / T^{2}$.

\section{3. $G_{2}$-Structures with Special Metrics Induced by Coupled Einstein Structures}

We can now use the coupled Einstein structure on $Z$ to construct a $G_{2}$-structure with full intrinsic torsion inducing an Einstein metric and a locally-conformal calibrated $G_{2}$-structure inducing a Ricci-flat metric.

First of all, we rescale the coupled Einstein structure on $Z$ in the following way:

$$
\begin{aligned}
\tilde{\omega} & =\frac{8}{5} \omega, \\
\tilde{\psi}_{+} & =\left(\frac{8}{5}\right)^{\frac{3}{2}} \psi_{+} .
\end{aligned}
$$

Then, $\left(\tilde{\omega}, \tilde{\psi}_{+}\right)$is a coupled structure with coupled constant $c=-\sqrt{10}$ and inducing the metric $\tilde{h}=\frac{8}{5} h$. $\operatorname{Moreover}, \operatorname{Scal}(\tilde{h})=30$ and $\operatorname{Ric}(\tilde{h})=5 \tilde{h}$. 
As we observed in Section 2, starting from the coupled Einstein structure $\left(\tilde{\omega}, \tilde{\psi}_{+}\right)$, we can construct a $G_{2}$-structure $\varphi$ on the sin-cone $S(Z)$ inducing the sin-cone metric $g_{\varphi}=d t^{2}+\sin ^{2}(t) \tilde{h}$. By Proposition 2, we then have that $g_{\varphi}$ is Einstein with Einstein constant 6 . Moreover, it is not difficult to show that the intrinsic torsion forms of the $G_{2}$-structure induced on the sin-cone by a coupled structure with coupled constant $c$ are:

$$
\begin{aligned}
\tau_{0} & =\frac{8 c+4}{7} \\
\tau_{1} & =\left(1-\frac{c}{3}\right) \cot (t) d t \\
\tau_{2} & =-\frac{\sin (2 t)}{2} w_{2}^{-} \\
\tau_{3} & =\frac{c-3}{7}\left(\sin ^{4}(t) \psi_{-}-\sin ^{3}(t) \cos (t) \psi_{+}+\frac{4}{3} \sin ^{2}(t) d t \wedge \omega\right)-\sin ^{2}(t) d t \wedge w_{2}^{-}
\end{aligned}
$$

Thus, the coupled Einstein structure $\left(\tilde{\omega}, \tilde{\psi}_{+}\right)$induces a $G_{2}$-structure with full intrinsic torsion and Einstein metric on the sin-cone $S(Z)$.

If we consider the $G_{2}$-structure $\varphi$ induced on the cone $C(Z)$ by $\left(\tilde{\omega}, \tilde{\psi}_{+}\right)$, then the metric $g_{\varphi}=d t^{2}+t^{2} \tilde{h}$ is Ricci-flat by Proposition 1. Moreover, the non-vanishing intrinsic torsion forms of the $G_{2}$-structure constructed on the cone from a coupled structure with coupled constant $c$ are:

$$
\begin{aligned}
& \tau_{1}=\left(\frac{1}{t}-\frac{1}{3 t} c\right) d t \\
& \tau_{2}=-t w_{2}^{-} .
\end{aligned}
$$

Therefore, the coupled Einstein structure $\left(\tilde{\omega}, \tilde{\psi}_{+}\right)$induces a locally-conformal calibrated $G_{2}$-structure on the cone $C(Z)$ whose associated metric is Ricci-flat.

Remark 4. It is worth observing here that calibrated $G_{2}$-structures inducing a Ricci-flat metric are actually parallel [26]. The previous example shows that a result of this kind is not true anymore for locally-conformal calibrated $G_{2}$-structures.

\section{Flows}

In this section, we study the behavior of coupled structures with respect to known evolution equations (flows) of SU(3)-structures.

The Hitchin flow, introduced in [19] as the Hamiltonian flow of a certain functional, allows one to construct (non-complete) metrics with holonomy in $G_{2}$ starting from a suitable $\mathrm{SU}(3)$-structure. The idea is to consider a six-manifold $N$ endowed with an $\mathrm{SU}(3)$-structure $\left(\omega, \psi_{+}\right)$and to define a $G_{2}$-structure on $M:=I \times N$ for some interval $I \subseteq \mathbb{R}$ by:

$$
\varphi=d t \wedge \omega+\psi_{+}
$$

where $\omega$ and $\psi_{+}$depend on the coordinate $t$ on $I$. If we require the $G_{2}$-structure to be parallel, we get that for each $t$ fixed, the $\mathrm{SU}(3)$-structure has to be half-flat and that, when $t$ is not fixed, the following evolution equations have to hold:

$$
\left\{\begin{array}{l}
\frac{\partial}{\partial t} \psi_{+}=d \omega \\
\frac{\partial}{\partial t} \omega \wedge \omega=-d \psi_{-}
\end{array} .\right.
$$


These equations are the so-called Hitchin flow equations. A solution of them with the initial condition a given $\mathrm{SU}(3)$-structure $\left(\omega(0), \psi_{+}(0)\right)$ exists when the latter is half-flat and analytic, but may not exist when the analytic hypothesis is dropped [40]. Moreover, it is easy to show that an $\mathrm{SU}(3)$-structure $\left(\omega(t), \psi_{+}(t)\right)$, which is half-flat for $t=0$ and evolves as prescribed in Equation (13), stays half-flat as long as it exists.

In the work [20], a generalization of the Hitchin flow was used to study the moduli space of $\mathrm{SU}(3)$-structure manifolds. The starting point to define this flow is to consider the embedding of an $\mathrm{SU}(3)$-structure into a non-compact manifold endowed with an integrable $G_{2}$-structure. This is motivated by the subject the authors are interested in, namely four-dimensional domain wall solutions of heterotic string theory that preserve $\mathcal{N}=\frac{1}{2}$ supersymmetry (see also [9]). In this case, the internal six-dimensional manifold is endowed with an $\mathrm{SU}(3)$-structure, and one can combine it with the direction perpendicular to the domain wall in the four-dimensional non-compact space time to get a seven-dimensional non-compact manifold endowed with a $G_{2}$-structure. The physical setting provides further constraints on the intrinsic torsion forms of the $G_{2}$-structure, which we will recall later. One can then study under which conditions a certain class of SU(3)-structures is preserved by this generalized flow.

It is then a natural question to ask whether the coupled condition is preserved by the Hitchin flow and, more generally, which constraints arise requiring that a solution of the generalized Hitchin flow is coupled as long as it exists. We begin giving the following definition.

Definition 14. Let $\left(\omega(t), \psi_{+}(t)\right)$ be a solution of the Hitchin flow defined on an interval $I \subseteq \mathbb{R}$ containing 0 and starting from a coupled structure at $t=0$. If $\left(\omega(t), \psi_{+}(t)\right)$ is a coupled structure for each $t \in I$, that is $d \omega(t)=c(t) \psi_{+}(t)$ for some smooth function $c: I \rightarrow \mathbb{R}$, we call it a coupled solution of the Hitchin flow.

Coupled solutions of the Hitchin flow can be easily characterized and induce an almost complex structure not depending on $t$.

Proposition 15. Let $N$ be a six-dimensional manifold, and suppose there exists on it a solution $\left(\omega(t), \psi_{+}(t)\right)$ of the Hitchin flow starting from a coupled structure $\left(\omega(0), \psi_{+}(0)\right)$ and defined on some interval $I \subseteq \mathbb{R}$ containing 0 . If $\left(\omega(t), \psi_{+}(t)\right)$ is a coupled solution, then there exists a smooth function $f: I \rightarrow \mathbb{R}$, such that

$$
\psi_{+}(t)=f(t) \psi_{+}(0)
$$

Conversely, if the pair $\left(\omega(t), \psi_{+}(t)\right)$ is a solution of the Hitchin flow with $\psi_{+}(t)=f(t) \psi_{+}(0)$, then it is a coupled solution.

Proof. If $\left(\omega(t), \psi_{+}(t)\right)$ is a solution of the Hitchin flow with $\psi_{+}(t)=f(t) \psi_{+}(0)$, then from the flow equation $\frac{\partial}{\partial t} \psi_{+}(t)=d \omega(t)$, we obtain:

$$
d \omega(t)=\frac{\partial}{\partial t}\left(f(t) \psi_{+}(0)\right)=\left(\frac{d}{d t} f(t)\right) \psi_{+}(0) .
$$


Thus, the solution is a coupled structure with $c(t)=\frac{d}{d t} f(t)$. Suppose now that the solution is coupled, $d \omega(t)=c(t) \psi_{+}(t)$. Then, from the flow equation, we obtain:

$$
\frac{\partial}{\partial t} \psi_{+}(t)=c(t) \psi_{+}(t)
$$

Working in local coordinates on $N$, it is easy to show that

$$
\psi_{+}(t)=f(t) \psi_{+}(0)
$$

where

$$
f(t)=e^{\int_{0}^{t} c(s) d s}
$$

Corollary 16. Let $\left(\omega(t), \psi_{+}(t)\right)$ be a coupled solution of the Hitchin flow on a six-dimensional manifold $N$. Then, the associated almost complex structure is $J(t)=J(0)$, that is it does not depend on $t$.

Proof. We know that $\psi_{+}(t)=f(t) \psi_{+}(0)$; therefore:

$$
J(t)=J_{\psi_{+}(t)}=J_{f(t) \psi_{+}(0)}=J_{\psi_{+}(0)}=J(0),
$$

since the almost complex structure induced by $\psi_{+}$does not change if we rescale $\psi_{+}$by a real constant.

\subsection{Coupled Solutions on Six-Dimensional Nilpotent Lie Algebras}

Working on six-dimensional nilpotent Lie algebras, it is possible to show that a coupled solution of the Hitchin flow may not exist. As we recalled in Proposition 11, the only six-dimensional nilpotent Lie algebras admitting a coupled structure are, up to isomorphisms, $\mathfrak{I}$ and $\mathfrak{N}$. In each case, with respect to the frame we considered, the pair $\left(\omega, \psi_{+}\right)$, where:

$$
\begin{aligned}
\omega & =e^{12}+e^{34}+e^{56}, \\
\psi_{+} & =e^{135}-e^{146}-e^{236}-e^{245},
\end{aligned}
$$

is a coupled structure with $d \omega=-\psi_{+}$. For completeness, we observe also that:

$$
\psi_{-}=J \psi_{+}=e^{136}+e^{145}+e^{235}-e^{246}
$$

The following result shows our claim.

Proposition 17. Consider the Hitchin flow on the six-dimensional nilpotent Lie algebras $\mathfrak{N}$ and $\mathfrak{I}$. Then, on $\mathfrak{I}$, there exists a coupled solution starting from Equation (14) at $t=0$, while on $\mathfrak{N}$, there are no coupled solutions of the Hitchin flow starting from Equation (14).

Proof. Let us start with $\mathfrak{I}$; it admits a coupled solution of the Hitchin flow already described in [41]. We recover it here starting from a suitable pair $\left(\omega(t), \psi_{+}(t)\right)$ and requiring that it satisfies the Hitchin flow equations. From Proposition 15, we know that $\left(\omega(t), \psi_{+}(t)\right)$ is a coupled solution if and only if:

$$
\psi_{+}(t)=f(t) \psi_{+}(0)=f(t)\left(e^{135}-e^{146}-e^{236}-e^{245}\right),
$$


with $f(0)=1$. It is also clear that $\psi_{-}(t)=f(t)\left(e^{136}+e^{145}+e^{235}-e^{246}\right)$. Moreover, since we already know the form of the solution, we consider three smooth functions $a_{1}(t), a_{2}(t), a_{3}(t)$ with $a_{i}(0)=1$ and such that:

$$
\omega(t)=a_{1}(t) e^{12}+a_{2}(t) e^{34}+a_{3}(t) e^{56} .
$$

From now on, we omit the $t$-dependence of the considered functions. The forms $\omega(t)$ and $\psi_{ \pm}(t)$ are compatible for each $t$, and from the normalization condition, we get:

$$
f^{2}=a_{1} a_{2} a_{3}
$$

From the first Hitchin flow equation in Equation (13), we obtain:

$$
\frac{d}{d t} f=-a_{3}
$$

while from the second one, we have:

$$
\begin{aligned}
\frac{d}{d t}\left(a_{1} a_{3}\right) & =0 \\
\frac{d}{d t}\left(a_{2} a_{3}\right) & =0 \\
\frac{d}{d t}\left(a_{1} a_{2}\right) & =-4 f .
\end{aligned}
$$

From Equations (17) and (18) and the starting conditions at $t=0$, we deduce that:

$$
a_{1}=a_{2}=\frac{1}{a_{3}}
$$

Using this result and Equation (15), it holds necessarily:

$$
f=\frac{1}{\sqrt{a_{3}}} .
$$

Thus, the ODE Equation (16) becomes:

$$
\frac{d}{d t} a_{3}=2 a_{3}^{2} \sqrt{a_{3}}
$$

and solving this, we get:

$$
a_{3}=(1-3 t)^{-\frac{2}{3}}
$$

It is then easy to check that also Equation (19) is satisfied. Then, the pair:

$$
\begin{aligned}
\omega(t) & =(1-3 t)^{\frac{2}{3}} e^{12}+(1-3 t)^{\frac{2}{3}} e^{34}+(1-3 t)^{-\frac{2}{3}} e^{56}, \\
\psi_{+}(t) & =(1-3 t)^{\frac{1}{3}}\left(e^{135}-e^{146}-e^{236}-e^{245}\right)
\end{aligned}
$$

is a coupled solution of the Hitchin flow.

We consider now $\mathfrak{N}$; we will show that there are no coupled solutions starting from Equation (14). In this case, we need:

$$
\psi_{+}(t)=f(t) \psi_{+}(0)=f(t)\left(e^{135}-e^{146}-e^{236}-e^{245}\right)
$$


with $f(0)=1$, while we consider 15 smooth real valued functions $b_{i j}(t), 1 \leq i<j \leq 6$, such that:

$$
\omega(t)=\sum_{1 \leq i<j \leq 6} b_{i j}(t) e^{i j}
$$

$b_{12}(0)=b_{34}(0)=b_{56}(0)=1$ and $b_{i j}(0)=0$ for the remaining functions. First of all, we impose that the equations resulting from the compatibility condition $\omega(t) \wedge \psi_{+}(t)=0$ are satisfied; then, we consider the first and the second Hitchin flow equation, and we compute the ODEs derived from them. What we obtain after solving some of these differential equations is that $f \equiv 0$, which cannot be possible.

\subsection{Generalized Hitchin Flow}

Since coupled solutions of the Hitchin flow may not exist in general, as Proposition 17 states, we can consider the generalized Hitchin flow and investigate which properties the intrinsic torsion forms have to satisfy in order to preserve the coupled condition.

In this case, we start with an $\mathrm{SU}(3)$-structure $\left(\omega, \psi_{+}\right)$depending on a parameter $t \in I \subseteq \mathbb{R}$, and we construct a $G_{2}$-structure on $M:=I \times N$ by:

$$
\varphi=\nu_{t} d t \wedge \omega+\Re(F \Psi)
$$

where $\nu_{t} \in C^{\infty}(M)$ and $F$ is a complex valued smooth function defined on $M$ and having constant module one. Observe that the Riemannian metric defined by $\varphi$ is:

$$
g_{\varphi}=\nu_{t}^{2} d t^{2}+h
$$

As we already recalled, in the case of $\mathcal{N}=\frac{1}{2}$ domain wall solutions, the non-vanishing intrinsic torsion forms of the $G_{2}$-structure are $\tau_{0}, \tau_{1}, \tau_{3}$. On $M=I \times N, \tau_{1}$ and $\tau_{3}$ can be decomposed as:

$$
\begin{aligned}
& \tau_{1}=u_{t} d t+\tau_{1}^{N}, \\
& \tau_{3}=d t \wedge \eta_{t}+\tau_{3}^{N},
\end{aligned}
$$

where $u_{t}$ is a smooth function on $M, \tau_{1}^{N}$ is a one-form on $N, \eta_{t}$ is a two-form on $N$ depending on $t$ and $\tau_{3}^{N}$ is a three-form on $N$. Moreover, the following constraints hold:

$$
\begin{aligned}
u_{t} & =\frac{1}{2} \frac{\partial}{\partial t} \phi, \\
\tau_{1}^{N} & =\frac{1}{2} d \phi, \\
d_{7} \tau_{0} & =0,
\end{aligned}
$$

where $\phi$ is the ten-dimensional dilaton, $d_{7}$ denotes the exterior derivative on $M$ and $d$ denotes it on $N$.

A general argument allows one to write down the equations of the $\mathrm{SU}(3)$-structure flow associated with the embedding and some relations between the torsion forms of the $\mathrm{SU}(3)$-structure and the $G_{2}$-structure. In particular:

$$
w_{4}=2 \tau_{1}^{N} .
$$

Therefore, if we have an $\mathrm{SU}(3)$-structure with vanishing $w_{4}$, we get $d \phi=2 \tau_{1}^{N}=0$. 
Following [20], we work in the gauge $F=1$, in this case:

$$
\varphi=\nu_{t} d t \wedge \omega+\psi_{+}
$$

If we suppose that the structure is coupled for each $t$, i.e.,

$$
\begin{aligned}
d \omega(t) & =c(t) \psi_{+}(t) \\
d \psi_{+}(t) & =0 \\
d \psi_{-}(t) & =-\frac{2}{3} c(t)(\omega(t))^{2}-w_{2}^{-}(t) \wedge \omega(t),
\end{aligned}
$$

where $c: I \rightarrow \mathbb{R}$ is a smooth function, such that $w_{1}^{-}(t)=-\frac{2}{3} c(t)$, then the two-form $\omega(t)$ evolves as:

$$
\frac{\partial}{\partial t} \omega(t)=\lambda_{t} \omega(t)+h_{t}
$$

where

$$
\begin{aligned}
\lambda_{t} & =2 u_{t}-\nu_{t} w_{1}^{-}(t), \\
h_{t} & =\nu_{t} w_{2}^{-}(t)-*\left(d \nu_{t} \wedge * \psi_{+}(t)\right) .
\end{aligned}
$$

Moreover, it follows from a general argument involving the flow equations that:

$$
d \lambda_{t}=0
$$

and using one of the constraints recalled earlier, we get:

$$
d u_{t}=\frac{1}{2} d\left(\frac{\partial}{\partial t} \phi\right)=\frac{1}{2} \frac{\partial}{\partial t}(d \phi)=0 .
$$

Taking the exterior derivative of both sides of Equation (22), we then have:

$$
d \nu_{t}=0
$$

Thus, $\nu_{t}$ is actually a function of $t$, and Equation (23) becomes $h_{t}=\nu_{t} w_{2}^{-}$.

Remark 5. With our convention, $w_{2}^{-}$here is $-w_{2}^{-}$in the work [20].

The flow equations for $\psi_{+}(t)$ and $\psi_{-}(t)$ determined in [20] reduce to the following in the coupled case:

$$
\begin{aligned}
\frac{\partial}{\partial t} \psi_{+}(t) & =\frac{3}{2} \lambda_{t} \psi_{+}(t)-\frac{7}{4} \tau_{0} \nu_{t} \psi_{-}(t)-\nu_{t} \gamma, \\
\frac{\partial}{\partial t} \psi_{-}(t) & =\frac{7}{4} \tau_{0} \nu_{t} \psi_{+}(t)+\frac{3}{2} \lambda_{t} \psi_{-}(t)+\nu_{t} J \gamma,
\end{aligned}
$$

where $\gamma$ is a primitive three-form of type $(2,1)+(1,2)$ appearing in the expression of the Hodge dual of $\tau_{3}^{N}$ on $N$.

We derive now all of the conditions that arise requiring these flow equations to preserve the coupled condition. We may sometimes omit the $t$-dependence of the forms for brevity.

First of all, suppose that for each $t$, the coupled condition $d \omega(t)=c(t) \psi_{+}(t)$ holds. Differentiating both sides with respect to $t$, we have:

$$
d\left(\frac{\partial}{\partial t} \omega\right)=\dot{c} \psi_{+}+c\left(\frac{3}{2} \lambda_{t} \psi_{+}-\frac{7}{4} \tau_{0} \nu_{t} \psi_{-}-\nu_{t} \gamma\right) .
$$


Moreover, taking the exterior derivative of both sides of Equation (21), using $d \nu_{t}=0$ and the hypothesis on the coupled condition, we obtain:

$$
d\left(\frac{\partial}{\partial t} \omega\right)=\lambda_{t} c \psi_{+}+\nu_{t} d w_{2}^{-} .
$$

Comparing the two equations, it follows:

$$
\nu_{t} d w_{2}^{-}=\dot{c} \psi_{+}+\frac{1}{2} c \lambda_{t} \psi_{+}-\frac{7}{4} c \tau_{0} \nu_{t} \psi_{-}-c \nu_{t} \gamma
$$

Wedging both sides by $\psi_{-}$and using the fact that $\gamma \wedge \psi_{-}=0$, since $\gamma \in \Lambda^{2,1} \oplus \Lambda^{1,2}$, we get:

$$
\nu_{t} d w_{2}^{-} \wedge \psi_{-}=\frac{2}{3} \dot{c} \omega^{3}+\frac{1}{3} c \lambda_{t} \omega^{3} .
$$

Since for each $t$ it holds $d w_{2}^{-} \wedge \psi_{-}=-\left|w_{2}^{-}\right|^{2} \frac{\omega^{3}}{6}$, where the norm is induced by $h(t)$, Equation (26) becomes:

$$
-\nu_{t}\left|w_{2}^{-}\right|^{2} \frac{\omega^{3}}{6}=\frac{2}{3} \dot{c} \omega^{3}+\frac{1}{3} c \lambda_{t} \omega^{3}
$$

and the following result is proven.

Proposition 18. Suppose that the generalized Hitchin flow preserves the coupled condition d $\omega(t)=$ $c(t) \psi_{+}(t)$. Then, the function $c(t)$ must evolve in the following way:

$$
\frac{\partial}{\partial t} c(t)=-\frac{1}{2} c(t) \lambda_{t}-\frac{1}{4} \nu_{t}\left|w_{2}^{-}(t)\right|_{h(t)}^{2} .
$$

Moreover, for each t, it must hold:

$$
d w_{2}^{-}=-\frac{1}{4}\left|w_{2}^{-}\right|^{2} \psi_{+}-\frac{7}{4} c \tau_{0} \psi_{-}-c \gamma .
$$

In order to preserve the closedness of $\psi_{+}(t)$, we need:

$$
d\left(\frac{\partial}{\partial t} \psi_{+}\right)=0
$$

Moreover, taking the exterior derivative of both sides of the flow Equation (24) of $\psi_{+}$, we have:

$$
d\left(\frac{\partial}{\partial t} \psi_{+}\right)=-\frac{7}{4} \tau_{0} \nu_{t} d \psi_{-}-\nu_{t} d \gamma
$$

Comparing the two equations, it then follows:

$$
d \gamma=-\frac{7}{4} \tau_{0} \nu_{t} d \psi_{-}
$$

Observe now that $d \gamma \wedge \omega=0$, since $\gamma$ is a primitive form of type $(2,1)+(1,2)$. Therefore, wedging both sides of Equation (27) by $\omega$ and recalling that $d \psi_{-} \wedge \omega=-\frac{2}{3} c \omega^{3}$, we get:

$$
\tau_{0} \nu_{t} c=0
$$

and then $\tau_{0}=0$, since both $c$ and $\nu_{t}$ cannot be zero. In particular:

$$
d \gamma=0
$$

We can summarize the results in the following: 
Proposition 19. If the closedness of $\psi_{+}$is preserved by the generalized Hitchin flow, then the intrinsic torsion form $\tau_{0}$ vanishes, and the thee-form $\gamma$ is closed.

Let us now consider the expression of $d \psi_{-}$in Equation (20) and differentiate it with respect to $t$, having in mind the results already obtained:

$$
d\left(\frac{\partial}{\partial t} \psi_{-}\right)=\left(-\frac{2}{3} \dot{c}-\frac{4}{3} c \lambda_{t}\right) \omega^{2}+\left(-\frac{4}{3} c \nu_{t}-\lambda_{t}\right) w_{2}^{-} \wedge \omega-\frac{\partial}{\partial t} w_{2}^{-} \wedge \omega-\nu_{t} w_{2}^{-} \wedge w_{2}^{-} .
$$

Taking the exterior derivative of both sides of the flow Equation (25) of $\psi_{-}(t)$, we get:

$$
d\left(\frac{\partial}{\partial t} \psi_{-}\right)=-\lambda_{t} c \omega^{2}-\frac{3}{2} \lambda_{t} w_{2}^{-} \wedge \omega+\nu_{t} d(J \gamma)
$$

Comparing the two equations, we obtain that the flow of $w_{2}^{-}$must obey the following equation:

$$
\frac{\partial}{\partial t} w_{2}^{-} \wedge \omega=\frac{1}{6} \nu_{t}\left|w_{2}^{-}\right|^{2} \omega^{2}+\left(-\frac{4}{3} c \nu_{t}+\frac{1}{2} \lambda_{t}\right) w_{2}^{-} \wedge \omega-\nu_{t} w_{2}^{-} \wedge w_{2}^{-}-\nu_{t} d(J \gamma)
$$

We also know that the following necessary conditions deriving from the Bianchi identity $d_{7} \hat{H}=0$ must hold:

$$
\begin{aligned}
d S^{X} & =0 \\
d S_{t} & =\frac{\partial}{\partial t} S^{X}
\end{aligned}
$$

where $\hat{H}=d t \wedge S_{t}+S^{X}$ is the component of the ten-dimensional flux along $M$.

Remark 6. The other constraint obtained from the Bianchi identities was recalled earlier; it is $d_{7} \tau_{0}=0$.

Using the previous results, it follows from [20] that for a coupled structure:

$$
S^{X}=\nu_{t}^{-1} u_{t} \psi_{-}+J \gamma, \quad S_{t}=0
$$

From the first identity Equation (28), we then get:

$$
d(J \gamma)=-\nu_{t}^{-1} u_{t} d \psi_{-}
$$

Observe that $d(J \gamma) \wedge \omega=0$. Thus, if we wedge both sides of Equation (30) by $\omega$, we obtain:

$$
\nu_{t}^{-1} u_{t} c=0
$$

from which follows $u_{t}=0$ and, as a consequence, $d(J \gamma)=0$. The second identity Equation (29) then reads:

$$
\frac{\partial}{\partial t}(J \gamma)=0
$$

We can summarize here some of the results obtained:

(i) The only non-vanishing intrinsic torsion form of the $G_{2}$-structure after imposing all conditions is $\tau_{3}$. Moreover, $* \tau_{3}^{X}=\gamma$ and $\eta_{t}=0$. 
(ii) $d \nu_{t}=0$.

(iii) $d \gamma$ and $d(J \gamma)=0$; thus, $\gamma$ is harmonic.

(iv) $\lambda_{t}=\frac{2}{3} \nu_{t} c(t)$.

In particular, the evolution equations of the differential forms defining the coupled structure become:

$$
\begin{aligned}
\frac{\partial}{\partial t} \omega(t) & =\frac{2}{3} \nu_{t} c(t) \omega(t)+\nu_{t} w_{2}^{-}(t), \\
\frac{\partial}{\partial t} \psi_{+}(t) & =\nu_{t} c(t) \psi_{+}(t)-\nu_{t} \gamma, \\
\frac{\partial}{\partial t} \psi_{-}(t) & =\nu_{t} c(t) \psi_{-}(t)+\nu_{t} J \gamma .
\end{aligned}
$$

Moreover, the intrinsic torsion forms of the coupled structure must evolve as:

$$
\begin{aligned}
\frac{\partial}{\partial t} c(t) & =-\frac{1}{3} \nu_{t}(c(t))^{2}-\frac{1}{4} \nu_{t}\left|w_{2}^{-}(t)\right|_{h(t)}^{2}, \\
\frac{\partial}{\partial t} w_{2}^{-}(t) \wedge \omega(t) & =\frac{1}{6} \nu_{t}\left|w_{2}^{-}(t)\right|_{h(t)}^{2}(\omega(t))^{2}-\nu_{t} c(t) w_{2}^{-}(t) \wedge \omega(t)-\nu_{t}\left(w_{2}^{-}(t)\right)^{2},
\end{aligned}
$$

and for each $t$, it must hold:

$$
d w_{2}^{-}=-\frac{1}{4}\left|w_{2}^{-}\right|^{2} \psi_{+}-c \gamma
$$

\subsection{The Hitchin Flow as a Particular Case of the Generalized Hitchin Flow}

If we suppose that $\nu_{t}=1$ and $\gamma=0$, then:

$$
\varphi=d t \wedge \omega+\psi_{+}
$$

is a parallel $G_{2}$-structure. In this case, the evolution equations of the differential forms $\omega(t), \psi_{+}(t), \psi_{-}(t)$ read:

$$
\begin{aligned}
\frac{\partial}{\partial t} \omega(t) & =\frac{2}{3} c(t) \omega(t)+w_{2}^{-}(t), \\
\frac{\partial}{\partial t} \psi_{+}(t) & =c(t) \psi_{+}(t), \\
\frac{\partial}{\partial t} \psi_{-}(t) & =c(t) \psi_{-}(t),
\end{aligned}
$$

the evolution equations of the intrinsic torsion forms of the coupled structure must be:

$$
\begin{aligned}
\frac{\partial}{\partial t} c(t) & =-\frac{1}{3}(c(t))^{2}-\frac{1}{4}\left|w_{2}^{-}(t)\right|_{h(t)}^{2} \\
\frac{\partial}{\partial t} w_{2}^{-}(t) \wedge \omega(t) & =\frac{1}{6}\left|w_{2}^{-}(t)\right|_{h(t)}^{2}(\omega(t))^{2}-c(t) w_{2}^{-}(t) \wedge \omega(t)-\left(w_{2}^{-}(t)\right)^{2},
\end{aligned}
$$

and for each $t$, the two-form $w_{2}^{-}$has to satisfy the following property:

$$
d w_{2}^{-}=-\frac{1}{4}\left|w_{2}^{-}\right|^{2} \psi_{+},
$$

which is one of the conditions widely discussed in Section 3. 
A solution of these equations, which is coupled for each $t$, is also a coupled solution of the Hitchin flow equations and vice versa. For example, the coupled solution of the Hitchin flow on the Iwasawa-Lie algebra $\mathfrak{I}$ obtained in the proof of Proposition 17 satisfies Equations (31), (32) and (33). In the general case, the presence of $w_{2}^{-}(t)$ in the flow equations makes rather complicated any attempt to solve them. However, we can show that a solution of them starting from a coupled SU(3)-structure stays coupled as long as it exists.

Proposition 20. Let $\left(\omega(t), \psi_{+}(t), c(t), w_{2}^{-}(t)\right)$ be a solution of Equations (31), (32) and (33), with the initial condition a coupled structure $\left(\omega(0), \psi_{+}(0)\right)$ satisfying $d \omega(0)=c(0) \psi_{+}(0)$. Then, $\left(\omega(t), \psi_{+}(t)\right)$ is a coupled structure as long as it exists.

Proof. Consider $d \omega(t)-c(t) \psi_{+}(t)$; differentiating with respect to $t$ and using the hypothesis, we get (omitting the $t$-dependence for brevity):

$$
\begin{aligned}
\frac{\partial}{\partial t}\left(d \omega-c \psi_{+}\right) & =d\left(\frac{\partial}{\partial t} \omega\right)-\dot{c} \psi_{+}-c \frac{\partial}{\partial t} \psi_{+} \\
& =\frac{2}{3} c d \omega+d w_{2}^{-}+\frac{1}{3} c^{2} \psi_{+}+\frac{1}{4}\left|w_{2}^{-}\right|^{2} \psi_{+}-c^{2} \psi_{+} \\
& =\frac{2}{3} c\left(d \omega-c \psi_{+}\right) .
\end{aligned}
$$

Thus, if we denote by $\rho(t)=d \omega(t)-c(t) \psi_{+}(t)$, we have that $\frac{\partial}{\partial t} \rho(t)=\frac{2}{3} c(t) \rho(t)$. Therefore, $\rho(t)=$ $q(t) \rho(0)$, where $q(t)=e^{\int_{0}^{t} \frac{2}{3} c(s) d s}$. However, $\rho(0)=d \omega(0)-c(0) \psi_{+}(0)=0$, since $\left(\omega(0), \psi_{+}(0)\right)$ is coupled. Then, $0=\rho(t)=d \omega(t)-c(t) \psi_{+}(t)$, and as a consequence, $d \psi_{+}(t)=0$.

\section{Conclusions}

In this paper, we considered from the mathematical point of view the properties of $\mathrm{SU}(3)$-structures, which are of interest in the case of $\mathcal{N}=1$ compactifications of Type IIA string theory to four-dimensional anti-de Sitter space on six-manifolds endowed with an SU(3)-structure, namely coupled structures satisfying (all or in part) the constraints given in Equations (4) and (5).

First of all, we derived some properties of such structures and some constraints implied by them. These need to be taken into account when one looks for explicit examples.

We then turned our attention to examples of six-manifolds endowed with this kind of SU(3)-structure. In the case of nilmanifolds, we already knew that up to isomorphisms, there are two non-abelian nilpotent Lie algebras admitting a coupled structure from [14]. Here, we showed that for only one of these, Equation (4) is satisfied, while Equation (5) cannot be ever satisfied. However, since in the physical setting, Equations (4) and (5) can be relaxed in the presence of sources, the nilmanifolds generated by $\mathfrak{I}$ and $\mathfrak{N}$ may be used to construct examples of the considered type of compactification. This was done for the Iwasawa manifold in [11]; thus, it would be interesting to see what happens for the nilmanifold corresponding to $\mathfrak{N}$. We also recalled an example firstly described in [6]; this is of particular interest, not only because it answers a question arising from [16], but also because it allows one to construct examples of $G_{2}$-structures with torsion inducing remarkable metrics.

In the last section, we considered the behavior of the coupled condition with respect to the Hitchin flow and one of its possible generalizations determined starting from four-dimensional domain wall 
solutions of heterotic string theory preserving $\mathcal{N}=\frac{1}{2}$ supersymmetry. We observed that it is not always possible to find coupled solutions of the Hitchin flow by working on explicit examples and derived the conditions implied by requiring that the coupled condition is preserved by the generalized Hitchin flow. An interesting open question would be to see whether there exist any example of a one-parameter family of SU(3)-structures that solves the Hitchin flow equations and is coupled for at least one, but not for all $t$.

\section{Acknowledgments}

The authors would like to thank Thomas Madsen for useful conversations. The authors are supported by the Project Progetti di Ricerca di Interesse Nazionale (PRIN) "Varietà reali e complesse: geometria, topologia e analisi armonica", by the Project Fondo per gli Investimenti della Ricerca di Base (FIRB) "Geometria Differenziale e Teoria Geometrica delle Funzioni”, and by Gruppo Nazionale per le Strutture Algebriche, Geometriche e le loro Applicazioni (GNSAGA) of Istituto Nazionale di Alta Matematica (INdAM).

\section{Author Contributions}

The authors collaborated during the research work and wrote the paper.

\section{Conflicts of Interest}

The authors declare no conflict of interest.

\section{References}

1. Strominger, A. Superstrings with Torsion. Nucl. Phys. B 1986, 274, 2534-284.

2. Gurrieri, S.; Lukas, A.; Micu, A. Heterotic string compactified on half-flat manifolds. Phys. Rev. D 2004, 70, 126009, doi:10.1103/PhysRevD.70.126009.

3. Lüst, D.; Tsimpis, D. Supersymmetric $\mathrm{AdS}_{4}$ compactifications of IIA supergravity. J. High Energy Phys. 2005, 027, doi:10.1088/1126-6708/2005/02/027.

4. Gurrieri, S. Compactifications on half-flat manifolds. Fortschr. Phys. 2005, 53, 278-336.

5. De Carlos, B.; Micu, A.; Gurrieri, S.; Lukas, A. Moduli stabilisation in heterotic string compactifications. J. High Energy Phys. 2006, doi: 10.1088/1126-6708/2006/03/005.

6. Tomasiello, A. New string vacua from twistor spaces. Phys. Rev. D 2008, 78, doi:10.1103/ PhysRevD.78.046007.

7. Klaput, M.; Lukas, A.; Matti, C. Bundles over Nearly-Kähler Homogeneous Spaces in Heterotic String Theory. J. High Energy Phys. 2011, 100, 1-34.

8. Lukas, A.; Matti, C. G-structures and Domain Walls in Heterotic Theories. J. High Energy Phys. 2011, 151, 1-31.

9. Gray, J.; Larfors, M.; Lüst, D. Heterotic domain wall solutions and SU(3) structure manifolds. J. High Energy Phys. 2012, 8, 1-37.

10. Larfors, M. Revisiting toric SU(3) structures. Fortschr. Phys. 2013, 61, 1031-1055. 
11. Caviezel, C.; Koerber, P.; Körs, S.; Lüst, D.; Tsimpis, D.; Zagermann, M. The effective theory of type IIA $\mathrm{AdS}_{4}$ compactifications on nilmanifolds and cosets. Class. Quantum Gravity 2009, 26, doi:10.1088/0264-9381/26/2/025014.

12. Koerber, P.; Lüst, D.; Tsimpis, D. Type IIA $\mathrm{AdS}_{4}$ compactifications on cosets, interpolations and domain walls. J. High Energy Phys. 2008, 38, doi:10.1088/1126-6708/2008/07/017.

13. Salamon, S. A tour of exceptional geometry. Milan J. Math. 2003, 71, 59-94.

14. Fino, A.; Raffero, A. Einstein Locally Conformal Calibrated $G_{2}$-structures. 2013, arXiv:1303.6137. arXiv.org e-Print archive. Available online: http://arxiv.org/abs/1303.6137 (accessed on 8 May 2015).

15. Madsen, T.B.; Salamon, S. Half-flat structures on $S^{3} \times S^{3}$. Ann. Glob. Anal. Geom. 2013, 44, 369-390.

16. Raffero, A. Half-flat structures inducing Einstein metrics on homogeneous spaces. Ann. Glob. Anal. Geom. 2015, doi:10.1007/s10455-015-9457-1.

17. Butruille, J.-B. Classification des variétés approximativement kähleriennes homogènes. Ann. Glob. Anal. Geom. 2005, 27, 201-225.

18. Foscolo, L.; Haskins, M. New $G_{2}$ holonomy cones and exotic nearly Kähler structures on the 6-sphere and the product of a pair of 3-spheres. 2015, arXiv:1501.07838. arXiv.org e-Print archive. Available online: http://arxiv.org/abs/1501.07838 (accessed on 8 May 2015).

19. Hitchin, N. Stable forms and special metrics. In Global Differential Geometry: The Mathematical Legacy of Alfred Gray; American Mathematical Society: Providence, RI, USA, 2001; pp. 70-89.

20. De la Ossa, X.; Larfors, M.; Svanes, E.E. Exploring SU(3)-Structure Moduli Spaces with Integrable $G_{2}$ Structures. 2014, arXiv:1409.7539. arXiv.org e-Print archive. Available online: http://arxiv.org/abs/1409.7539 (accessed on 8 May 2015).

21. Reichel, W. Über die Trilinearen Alternierenden Formen in 6 und 7 Veränderlichen. Ph.D. Thesis, Ernst-Moritz-Arndt-Universität Greifswald, Greifswald, Germany, 1907.

22. Hitchin, N. The geometry of three-forms in six dimensions. J. Differ. Geom. 2000, 55, 547-576.

23. Chiossi, S.; Salamon, S. The intrinsic torsion of $\mathrm{SU}(3)$ and $G_{2}$ structures. 2002, arXiv:math/0202282. arXiv.org e-Print archive. Available online: http://arxiv.org/abs/math/ 0202282 (accessed on 8 May 2015).

24. Agricola, I.; Chiossi, S.; Friedrich, T.; Höll, J. Spinorial description of SU(3)- and $G_{2}$-manifolds. 2014, arXiv:1411.5663. arXiv.org e-Print archive. Available online: http://arxiv.org/ pdf/1411.5663.pdf (accessed on 8 May 2015).

25. Bedulli, L.; Vezzoni, L. The Ricci tensor of SU(3)-manifolds. J. Geom. Phys. 2007, 57, 1125-1146.

26. Bryant, R.L. Some Remarks on $G_{2}$-structures. 2003, arXiv:math/0305124. arXiv.org e-Print archive. Available online: http://arxiv.org/abs/math/0305124 (accessed on 8 May 2015).

27. Friedrich, T.; Ivanov, S. Parallel spinors and connections with skew-symmetric torsion in string theory. Asian J. Math. 2002, 6, 303-335.

28. Karigiannis, S.; McKay, B.; Tsui, M.-P. Soliton solutions for the Laplacian co-flow of some $\mathrm{G}_{2}$-structures with symmetry. Differ. Geom. Appl. 2012, 30, 318-333.

29. O'Neill, B. Semi-Riemannian Geometry, with Applications to Relativity; Academic Press: New York, NY, USA, 1983. 
30. Boyer, C.P.; Galicki, K. Sasakian geometry, holonomy, and supersymmetry. Handbook of pseudo-Riemannian geometry and supersymmetry. IRMA Lect. Math. Theor. Phys. Eur. Math. Soc. Zürich 2010, 16, 39-83.

31. Magnin, L. Sur les algèbres de Lie nilpotentes de dimension $\leq$ 7. J. Geom. Phys. 1986, 3, 119-144.

32. Malcev, A.I. On a class of homogeneous spaces; American Mathematical Society: Providence, RI, USA, 1951.

33. Milnor, J.: Curvatures of Left Invariant Metrics on Lie Groups. Adv. Math. 1976, 21, 293-329.

34. Abbena, E.; Garbiero, S.; Salamon, S. Hermitian geometry on the Iwasawa manifold. Boll. Unione Mat. Ital. B 1997, 11, 231-249.

35. Muškarov, O. Almost Hermitian structures on twistor spaces and their types. Atti Sem. Mat. Fis. Univ. Modena 1989, 37, 285-297.

36. Apostolov, V.; Grantcharov, G.; Ivanov, S. Hermitian structures on twistor spaces. Ann. Glob. Anal. Geom. 1998, 16, 291-308.

37. Eells, J.; Salamon, S. Twistorial construction of harmonic maps of surfaces into four-manifolds. Ann. Scuola Norm. Super. Pisa Cl. Sci. 1985, 12, 589-640.

38. Xu, F. Geometry of SU(3) manifolds. Ph.D. Thesis, Duke University, Durham, NC, USA, May 2008.

39. Besse, A. Einstein Manifolds. In Ergebnisse der Mathematik und ihrer Grenzgebietev; Springer: Berlin/Heidelberg, Germany, 1987.

40. Bryant, R.L. Non-Embedding and non-Extension Results in Special Holonomy. In The Many Facets of Geometry; Oxford University Press: Oxford, UK, 2010; pp. 346-367.

41. Chiossi, S.; Fino, A. Conformally parallel $G_{2}$ structures on a class of solvmanifolds. Math. Z. 2006, $252,825-848$.

(c) 2015 by the authors; licensee MDPI, Basel, Switzerland. This article is an open access article distributed under the terms and conditions of the Creative Commons Attribution license (http://creativecommons.org/licenses/by/4.0/). 